\title{
An Uncertain Programming Model for Fixed Charge Transportation Problem with After-Sale Service
}

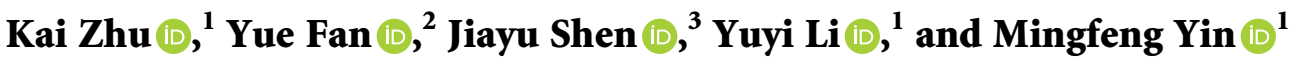 \\ ${ }^{1}$ School of Automobile and Traffic Engineering, Jiangsu University of Technology, Changzhou 213001, Jiangsu, China \\ ${ }^{2}$ The 723 Institute of CSIC, Yangzhou 225101, Jiangsu, China \\ ${ }^{3}$ Department of Public Basic Courses, Nanjing Vocational University of Industry Technology, Nanjing 210023, Jiangsu, China
}

Correspondence should be addressed to Kai Zhu; fatkyo@163.com

Received 8 August 2021; Revised 27 November 2021; Accepted 28 January 2022; Published 21 February 2022

Academic Editor: Sumit Chandok

Copyright ( 2022 Kai Zhu et al. This is an open access article distributed under the Creative Commons Attribution License, which permits unrestricted use, distribution, and reproduction in any medium, provided the original work is properly cited.

\begin{abstract}
This paper constructs an uncertain mathematical model for the fixed charge transportation problem in which several kinds of items are transported from different sources to various destinations via different methods. In addition, after-sale service such as product return and exchange that help to raise the customer's satisfaction is considered during the trade to meet the reality. Meanwhile, some indeterministic factors may occur during the transportation process; probability theory is not the best option due to the lack of adequate historical data. In the light of this statement, some parameters like supplies, demands, return rate, and exchange rate are all defined as uncertain variables to formulate the uncertain programming model. After that, the equivalent deterministic models are derived with the help of uncertainty theory. Finally, some numerical experiments are implemented via three algorithms; the optimal values are displayed and compared to show the application of this problem. The study of this paper provides an applicable method for the decision maker.
\end{abstract}

\section{Introduction}

Transportation has participated in human's daily life for quite a long period, which establishes closer connections between different persons and locations, facilitating the globalization. Hitchcock [1] put forward a typical transportation problem (TP) for the first time, which was later discussed in [2], but only two constraints like availability and demand were put into consideration. After that, Arsham and Kahn [3] created a refined linear programming method for the transportation problems and Cooper and Cooper [4] designed a solution method for a series of concave transportation problems on the premise of separable objective function. However, the real transportation process is more complex; thus, more aspects such as transportation time, mode, capacity, and so on need to be discussed to make the problem closer to the reality. In the light of this statement, Das et al. [5] created a programming approach for a multiobjective TP from the perspective of decision maker, which was illustrated via three possible cases. Aneja and Nair [6] studied a double criterion TP with relevant objectives in practical situations and developed a nondominated solution to minimize the total cost. Sun [7] considered exclusionary side constraints for a TP and established a programming model that was settled by traditional algorithms to find feasible integer solutions.

A special branch of the traditional TP is the fixed charge transportation problem (FCTP). Besides the direct transportation cost related to the product, an extra part called fixed charge based on the route independent of the transportation quantity is put into consideration. Moreover, in reality, many uncertain factors such as traffic flow or weather condition may occur during the transportation process, making the problems more complex. In this condition, this paper focuses on the fixed charge transportation problem under the uncertain environment, proposing the mathematical model and corresponding solution methods.

1.1. Literature Review. In the past several decades, many researchers have focused on the models and corresponding solution methods for various types of FCTPs. Since it was 
initiated in [8], many research studies began to study it with different considerations. Robers and Cooper [9] proposed a refined algorithm with adjacent extreme point technique to solve the FCTP. Farley and Richardson [10] studied a particular case of identical fixed charges during the transportation period. Sagratella et al. [11] introduced a noncooperative FCTP with several competing players and made discussions on three versions about it. A simple but effective heuristic procedure was designed in [12] to solve a series of small FCTPs, and the best solution was achieved via several steps. When coming to a two-stage FCTP that involved plants, customers, and depots, Calvete et al. [13] created an evolutionary algorithm-based algorithm to minimize the overall costs. A nonlinear factor was added for a FCTP with multiple transportation modes in crisp and interval environments [14], and a crossover and a mutation scheme was renewed for solving the numerical examples.

The above researches about transportation problem are all within the deterministic framework, in which the parameters are deemed to be crisp values. However, indeterminacy tends to occur during the transportation process; thus, some parameters such as supplies, demands, and transportation time are not suitable to be discussed in indeterministic scenarios. Yang and Feng [15] studied a bicriteria solid TP in stochastic environment and constructed three mathematical models under different criteria for this problem. A stochastic multistage FCTP was designed in [16], where a time-limited demand was met by the producer. In 1965, Zadeh [17] created fuzzy set theory that was later applied in several aspects $[18,19]$, and many scholars made further investigations about FCTP in fuzzy environment. Yang and Liu [20] treated several parameters as fuzzy variables in a FCTP and studied the problem under three criteria with the help of credibility theory. A multiobjective FCTP with intuitionistic fuzzy data was studied in [21], which was then transformed into interval-valued problem. There exist more relevant research studies in indeterministic situations [22, 23].

All these studies with stochastic or fuzzy variables make the problem more realistic, which are under the framework of probability theory and fuzzy theory. When some uncertain factors occur, the assumption of random or fuzzy distribution depends on the adequate sample size. However, there exist technical difficulties or economic reasons, which prevent sufficient experimental or observed data from being obtained. Under this situation, an alternative option is to rely on the belief degrees evaluated by domain experts. Nevertheless, there exists a big shortcoming in belief degree that some counterintuitive conclusions may occur in some occasions, which leads to improper assessments. In order to get rid of such problems, uncertainty theory [24] and its refined edition [25] came into being. Since then, it has gained its popularity in massive fields [26-30], in which transportation is an important section. A typical TP was discussed in [31] where truck times and transportation costs were supposed to follow uncertain distributions, and a multiobjective optimization model was created. For a typical Solid TP, two kinds of programming models under two criteria were constructed in [32] with the help of uncertainty theory. An uncertain multiobjective multi-item FCTP with budget constraint was studied in [33], and three different models under different criteria were designed. For other FCTPs, similar methods were applied in [34-38] with different applications.

1.2. Research Gap and the Proposed Contribution. As mentioned in Section 1.1, there exist plenty of transportation problems that focus on several aspects such as transportation time, capacity, damage rate, and so on. However, in realworld scenarios, people will absolutely care about their benefits. For example, defective products may be sold to the customers due to defective rate, which would be unacceptable. At this moment, an exchange option will be made. Besides, impulsive purchasing always occurs around us, and product return policy must be available to guarantee the customer's benefits according to relative regulations. Thus, under this circumstance, after-sale service such as item return and item exchange needs to been considered in order to facilitate the service quality. Based on the normal transportation problem, this paper studies a FCTP in which customer's extra demand (return or exchange) is satisfied. As the manager has to afford the transportation cost, how to schedule a plan to minimize the cost becomes a challenge. Besides, in reality, their always exit some indeterministic factors during the transportation. To make this problem more realistic, this paper puts uncertain factors into this FCTP, establishing an uncertain programming model with the help of uncertainty theory, where after-sale service is particularly considered.

Compared with the existing literature, the main contributions of this paper are organized as follows. This paper considers a FCTP with extra costs brought by after-sales services, and an uncertain model is built with some uncertain constraints. Then, the original programming model is extended under the expected value criterion and chanceconstrained criterion, and corresponding deterministic forms are built with the help of uncertainty theory. After that, three kinds of algorithms are selected to solve the numerical examples, and their performances are compared. Moreover, we alter the original model by adding nonlinear elements to make further comparisons.

1.3. Managerial Insights Based on the Research. This paper considers the after-sale service in the transportation problem; the goal to minimize the total transportation cost is realized under the uncertain circumstance. On the one hand, after-sale service such as product return and exchange is essential, which helps to refine service quality and customer's satisfaction. However, this part has not been considered yet. On the other hand, some indeterministic factors will occur everywhere, including the transportation process, causing the problem more complex. Generally, we prefer to describe the indeterminacy with probability. However, it is not always reasonable to use probability theory to illustrate all the indeterminacies as it requires adequate information. Actually, low frequency events will occur in our daily life; at this moment, uncertainty theory takes its effect. Under this 
circumstance, how to schedule a proper transportation plan to lower the total costs is a challenge. The study in this paper provides an applicable method for the decision maker so that he can deal with some uncertain factors without losing reliability from customers. This research could also be extended to other aspects in which decision/plan needs to be made within indeterministic condition.

The structure of the remainder of this paper is organized as follows. Section 2 illustrates some basic information about uncertainty theory, and Section 3 elaborates the transportation problem. In Section 4, expected value programming model and chance-constrained model are proposed, and their equivalent forms with determinacy are deducted in Section 5. Section 6 exhibits the applications of this uncertain model via several numerical experiments.

\section{Preliminary}

As the uncertain theory is utilized in this paper, some basic concepts about it should be introduced before establishing the mathematical model.

Suppose $\mathscr{L}$ is a $\sigma$-algebra over a nonempty set $\Gamma$. Each event depicted by element $\Lambda \in \mathscr{L}$ with a value of $\mathscr{M}\{\Lambda\}$ labels the chance that uncertain event $\Lambda$ occurs. A set function $\mathscr{M}$ from $\mathscr{L}$ to $[0,1]$ is called an uncertain measure if it satisfies the axioms of normality, duality, and subadditivity [24].

Then, if a variable is treated as an uncertain one, its uncertainty distribution is defined as follows [25]:

$$
\mathscr{M}\{\xi \leq x\}=\Phi(x) .
$$

Example 1. An uncertain variable $\xi$ follows a normal uncertainty distribution:

$$
\Phi(x)=\left(1+\exp \left(\frac{\pi(e-x)}{\sqrt{3} \sigma}\right)\right)^{-1}, \quad x \in \mathfrak{R},
$$

where $e$ and $\sigma$ are real numbers with $\sigma>0$. Then, the variable $\xi$ is named as a normal uncertain variable expressed as $\xi \sim \mathcal{N}(e, \sigma)$.

Example 2. A variable is called linear if it follows linear uncertain distribution:

$$
\Phi(x)= \begin{cases}0, & \text { if } x \leq a, \\ \frac{x-a}{b-a}, & \text { if } a \leq x \leq b, \\ 1, & \text { if } x \geq b,\end{cases}
$$

where $0<a<b$, and it is denoted as $\xi \sim \mathscr{L}(a, b)$.

Definition 1 (see [24]). An uncertain distribution $\Phi(x)$ is said to be regular if its inverse function $\Phi^{-1}(x)$ exists and is unique for each $\alpha \in(0,1)$. Then, the inverse function $\Phi^{-1}$ is called the inverse uncertainty distribution of $\xi$.
Then, for the normal distribution $\xi \sim \mathcal{N}(e, \sigma)$ in $\mathrm{Ex}-$ ample 1, we get its inverse distribution function as

$$
\Phi^{-1}(\alpha)=e+\frac{\sigma \sqrt{3}}{\pi} \ln \frac{\alpha}{1-\alpha} .
$$

For the linear distribution $\mathscr{L}(a, b)$ in Example 2, the corresponding inverse distribution function can be expressed as

$$
\Phi^{-1}(\alpha)=(1-\alpha) a+\alpha b
$$

Definition 2 (see [24]). Let $\xi$ be an uncertain variable. Then, the expected value of $\xi$ is defined by

$$
E[\xi]=\int_{0}^{+\infty} \mathscr{M}\{\xi \geq r\} \mathrm{d} r-\int_{-\infty}^{0} \mathscr{M}\{\xi \leq r\} \mathrm{d} r
$$

on the premise that at least one of the two integrals is finite.

For simplicity, the expected value is defined as follows [25]:

$$
E[\xi]=\int_{0}^{1} \Phi^{-1}(\alpha) \mathrm{d} \alpha
$$

Theorem 1 (see [25]). Let $\xi_{1}, \xi_{2}, \ldots, \xi_{n}$ be independent regular uncertain variables with uncertainty distributions $\Phi_{1}, \Phi_{2}, \ldots, \Phi_{n}$, respectively. If the function is strictly increasing with respect to $x_{1}, x_{2}, \ldots, x_{m}, \xi=f\left(\xi_{1}, \xi_{2}, \ldots, \xi_{n}\right)$ is an uncertain variable with inverse uncertainty distribution $\Psi^{-1}(\alpha)=f\left(\Phi_{1}^{-1}(\alpha), \ldots, \Phi_{n}^{-1}(\alpha)\right)$

Then, based on equation (7), the expected value of uncertain variable $\xi=f\left(\xi_{1}, \xi_{2}, \ldots, \xi_{n}\right)$ can be described as follows [25]:

$$
E[\xi]=\int_{0}^{1} f\left(\Phi_{1}^{-1}(\alpha), \ldots, \Phi_{n}^{-1}(\alpha) \mathrm{d} \alpha,\right.
$$

as long as $E[\xi]$ exists.

Theorem 2 (see [25]). If $\xi$ and $\eta$ are independent uncertain variables with finite expected values, we have $E[a \xi+b \eta]=$ $a E[\xi]+b E[\eta]$ for any real numbers $a$ and $b$.

Based on the above theorems, some results related to uncertain distribution can be achieved [39].

$$
\begin{aligned}
E\left[\xi^{k}\right]= & \int_{0}^{1}\left(\Phi^{-1}(\alpha)\right)^{k} \mathrm{~d} \alpha \\
E\left[(\xi-E(\xi))^{k}\right]= & \int_{0}^{\infty}(1-\Phi(\sqrt[k]{x}-E[\xi])) \mathrm{d} x \\
& -\int_{-\infty}^{0} \Phi(\sqrt[k]{x}+E[\xi]) \mathrm{d} x .
\end{aligned}
$$

\section{Problem Description}

3.1. Assumptions. A FCTP with after-sale service under uncertain environment is analyzed. The products are available at several sources, which need to be transported via various 
conveyances to different destinations. To make it closer to actuality, return and exchange policies are available for customers. The manager's duty is to schedule a proper transportation plan that minimizes the total cost. To make the problem more explicit, we make the following assumptions:

(i) Items are carried from sources to destinations via different conveyances without any loss, which means no damageability will occur during the transportation.

(ii) Some customers plan to return or exchange the item after the purchase due to different reasons, which is fully supported by the policy. And the customer will receive the same amount of same item for exchange option.

(iii) All the returned items or exchanged items must be transported back to the manufacture at the retailer's expense before sold for the second time.

3.2. Notations. The research is to schedule an optimal transportation plan under some constraints to make the total transportation cost lowest. The following notations are described from three aspects as indices, parameters, and decision variable.

Indices:

$i$ : index for sources where products are available $(i=1,2, \ldots, M)$,

$j$ : index for destinations where products are conveyed $(j=1,2, \ldots, N)$,

$k$ : index for conveyances through which the products are transported $(k=1,2, \ldots, K)$.

Parameters:

$A_{i}$ : the maximum amount at source $i$,

$B_{j}$ : the minimum demand at destination $j$,

$V_{k}$ : the maximum transportation capacity of conveyance $k$,

$\lambda_{j}$ : return rate at destination $j \eta_{j}$ : exchange rate at destination $j$,

$c_{i j k}$ : direct transportation cost from source $i$ to destination $j$ via conveyance $k$,

$e_{j}$ : direct transportation cost from destination $j$ back to the manufacture,

$f_{i j k}$ : the fixed charge from source $i$ to destination $j$ via conveyance $k$.

Decision variables:

$x_{i j k}$ : the product qunantity carried from source it o destination $j$ via conveyancek.

3.3. Model Formulation. In this problem, the manager needs to afford two kinds of costs. The first one happens from sources to destinations, which includes direct cost $\sum_{i=1}^{M} \sum_{j=1}^{N} \sum_{k=1}^{K} c_{i j k} x_{i j k}$ and the fixed charge $\sum_{i=1}^{M} \sum_{j=1}^{N} \sum_{k=1}^{K} y_{i j k} f_{i j k}$, where

$$
y_{i j k}= \begin{cases}1, & \text { if } x_{i j k}>0, \\ 0, & \text { otherwise, }\end{cases}
$$

indicates that the manager has to pay for the fixed charge when conveyance $k$ from source $i$ to destination $j$ is occupied. The second one is the cost from destinations back to the manufacturer.

Based on the above assumptions and notations, the total transportation cost described as the objective function is

$$
\begin{aligned}
\mathscr{Z} & =\sum_{i=1}^{M} \sum_{j=1}^{N} \sum_{k=1}^{K} c_{i j k} x_{i j k}+\sum_{i=1}^{M} \sum_{j=1}^{N} \sum_{k=1}^{K} y_{i j k} f_{i j k}+\sum_{j=1}^{N}\left[e_{j}\left(\lambda_{j}+\eta_{j}\right) \sum_{i=1}^{M} \sum_{k=1}^{K} x_{i j k}\right] \\
& =\sum_{i=1}^{M} \sum_{j=1}^{N} \sum_{k=1}^{K}\left[c_{i j k} x_{i j k}+y_{i j k} f_{i j k}+e_{j}\left(\lambda_{j}+\eta_{j}\right) x_{i j k}\right],
\end{aligned}
$$

subjected to 


$$
\begin{aligned}
& \left\{\sum_{j=1}^{n} \sum_{k=1}^{l} x_{i j k} \leq A_{i},\right. \\
& \left\{\sum_{i=1}^{m} \sum_{k=1}^{l} x_{i j k} \geq\left(1+\eta_{j}\right) B_{j},\right. \\
& \left\{\sum_{i=1}^{m} \sum_{j=1}^{n} \sum_{p=1}^{q} x_{i j k} \leq V_{k},\right. \\
& \left\{x_{i j k} \geq 0 ; y_{i j k} \in\{0,1\}\right. \text {, }
\end{aligned}
$$

where $i=1,2, \ldots, M ; j=1,2, \ldots, N ; k=1,2, \ldots, K$; and $r=1,2, \ldots, R$, respectively.

The total cost is defined in the objective function equation (14) when after-sale service is considered. Both returned items and exchanged items increase the cost for transportation back to the manufacture. Besides, constraint equation (15) means that the inventory $A_{i}$ is sufficient to satisfy the total product amount carried from source $i$.

As we have made the assumption that due to some different reasons, some customers will return or change the items that were purchase previously. For the returned item, there is no need to increase the product amount, but for the customer who wants to make an exchange, an extra demand is required. As shown in constraint equation (16), an additional amount over its least demand $B_{j}$ should be put into consideration based on the exchange rate.

Constraint equation (17) implies that the capacity $V_{k}$ of conveyance $k$ should be no less than the total transportation amount.

Formula (18) denotes the variables definition constraints.

\section{Uncertain Mathematical Models}

As mentioned above, when sufficient historical data are accessible, we can easily calculate the distributions of the different parameters. However, the actuality is not always as what we expect. In this problem, we regard availability $\left(A_{i}\right)$, demand $\left(B_{j}\right)$, transportation capacity $\left(V_{k}\right)$, return rate $\left(\lambda_{j}\right)$, exchange rate $\left(\eta_{j}\right)$, unit transportation cost $\left(c_{i j k}\right)$, and fixed charge $\left(f_{i j k}\right)$ as uncertain variables and the rest as deterministic ones. To make the problem more clear, the uncertain parameters are rewritten in the uncertain forms as $\widetilde{A}_{i}, \widetilde{B}_{j}, \widetilde{V}_{k}, \widetilde{\lambda}_{j}, \widetilde{\eta}_{j}, \widetilde{c}_{i j k}$, and $\widetilde{f}_{i j k}$. Then, the problem description could be altered to

$$
\left\{\begin{array}{l}
\min [Z] \\
\text { s.t. } \\
\sum_{j=1}^{N} \sum_{k=1}^{K} x_{i j k} \leq \widetilde{A}_{i} \\
\sum_{i=1}^{M} \sum_{k=1}^{K} x_{i j k} \geq\left(1+\widetilde{\eta}_{j}\right) \widetilde{B}_{j} \\
\sum_{i=1}^{M} \sum_{j=1}^{N} x_{i j k} \leq \widetilde{V}_{k},
\end{array}\right.
$$

where

$$
Z=\sum_{i=1}^{M} \sum_{j=1}^{N} \sum_{k=1}^{K}\left[\widetilde{c}_{i j k} x_{i j k}+y_{i j k} \widetilde{f}_{i j k}+e_{j}\left(\widetilde{\lambda}_{j}+\widetilde{\eta}_{j}\right) x_{i j k}\right] .
$$

Definition 3. A feasible solution $x^{*}$ is called the expected optimal solution if

$$
E\left[f\left(x^{*}\right)\right] \geq E[f(x)]
$$

holds for any feasible solution $x$, where $f$ represents the objective function.

This criterion aims to minimize the expected value of objective function within several constraints. Thus, the corresponding model of equation (19) under this criterion could be described as follows:

$$
\left\{\begin{array}{l}
\min E[Z] \\
E\left[\sum_{j=1}^{N} \sum_{k=1}^{K} x_{i j k}-\widetilde{A}_{i}\right] \leq 0, \quad i=1,2, \ldots, M \\
E\left[\sum_{i=1}^{M} \sum_{k=1}^{K} x_{i j k}-\left(1+\widetilde{\eta}_{j}\right) \widetilde{B}_{j}\right] \geq 0, \quad j=1,2, \ldots, N \\
E\left[\sum_{i=1}^{M} \sum_{j=1}^{N} x_{i j k}-\widetilde{V}_{k}\right] \leq 0, \quad k=1,2, \ldots, K .
\end{array}\right.
$$

From another point of view, in reality, the manager will consider the risk when making transportation plan, which means the manager has to schedule an optimal transportation plan on the premise of a predetermined upper bound. Under this circumstance, when a confidence level $\alpha \in\{0,1\}$ is given, the manager is willing to achieve a smallest value $\bar{f}$ such that the uncertain variable $E\left[f\left(x^{*}\right)\right]$ is not more than $\bar{f}$, which leads to the following chance-constrained criterion.

Definition 4. A feasible solution $x^{*}$ is called $\alpha$-optimal solution if

$$
\min \left\{\bar{f} \mid \mathscr{M}\left\{f\left(x^{*}\right) \leq \bar{f}\right\} \geq \alpha\right\} \leq \max \{\bar{f} \mid \mathscr{M}\{f(x) \leq \bar{f}\} \geq \alpha\},
$$

holds for any feasible solution $x$, where $\alpha$ is a confidence level.

Thus, with the different confidence levels $\alpha$, the model of equation (19) under this case could be altered to

$$
\left\{\begin{array}{l}
\min \bar{Z} \\
\text { s.t. } \\
\mathscr{M}\{Z \leq \bar{Z}\} \geq \alpha \\
\mathscr{M}\left\{\sum_{j=1}^{N} \sum_{k=1}^{K} x_{i j k} \leq \widetilde{A}_{i}\right\} \geq \alpha_{i}, \quad i=1,2, \ldots, M \\
\mathscr{M}\left\{\left(1+\widetilde{\eta}_{j}\right) \widetilde{B}_{j} \leq \sum_{i=1}^{M} \sum_{k=1}^{K} x_{i j k}\right\} \geq \beta_{j}, \quad j=1,2, \ldots, N \\
\mathscr{M}\left\{\sum_{i=1}^{M} \sum_{j=1}^{N} x_{i j k} \leq \tilde{V}_{k}\right\} \geq \gamma_{k}, \quad k=1,2, \ldots, K,
\end{array}\right.
$$


where $\alpha, \alpha_{i}(i=1,2, \ldots, M), \beta_{j}(j=1,2, \ldots, N)$, and $\gamma_{k}(k=$ $1,2, \ldots, K)$ are prespecified confidence levels.

\section{Deterministic Transformations}

Obviously, there exist some uncertain variables that will bring difficulty for the computing and simulation. To simplify the calculation process, a natural option is to transform the uncertain models into deterministic ones, which is fully supported by the uncertain theory. The transformation of the equivalent deterministic forms is explicated in this section.

Theorem 3. We assume that the independent uncertain variables $\widetilde{A}_{i}, \widetilde{B}_{j}, \widetilde{V}_{k}, \widetilde{\lambda}_{j}, \widetilde{\eta}_{j}, \widetilde{c}_{i j k}$, and $\widetilde{f}_{i j k}$ have corresponding uncertainty distributions as $\Phi_{\widetilde{A}_{i}}, \Phi_{\widetilde{B}_{j}}, \Phi_{\widetilde{V}_{k}}, \Phi_{\tilde{\lambda}_{j}}, \Phi_{\widetilde{\eta}_{j}}, \Phi_{\widetilde{c}_{i j k}}$, and $\Phi \sim_{f_{i j k}}$, respectively, and then the model in equation (22) will have the following deterministic description:

$$
\begin{aligned}
& \left\{\min \sum_{i=1}^{M} \sum_{j=1}^{N} \sum_{k=1}^{K}\left\{\begin{array}{c}
x_{i j k} \int_{0}^{1} \Phi_{\tilde{c}_{i j k}}^{-1}(\alpha) \mathrm{d} \alpha+y_{i j k} \int_{0}^{1} \Phi_{\tilde{f}_{i j k}^{-1}}^{-1}(\alpha) \mathrm{d} \alpha \\
+e_{j}\left[\int_{0}^{1} \Phi_{\tilde{\lambda}_{j}}^{-1}(\alpha) \mathrm{d} \alpha+\int_{0}^{1} \Phi_{\bar{\eta}_{j}}^{-1}(\alpha) \mathrm{d} \alpha\right] x_{i j k}
\end{array}\right\},\right. \\
& \left\{\sum_{j=1}^{N} \sum_{k=1}^{\text {s.t. }} x_{i j k}-\int_{0}^{1} \Phi_{\widetilde{A}_{i}}^{-1}(1-\alpha) \mathrm{d} \alpha \leq 0,\right. \\
& \left\{\int_{0}^{1}\left[1+\Phi_{\tilde{\eta}_{j}}^{-1}(\alpha)\right] \Phi_{\widetilde{B}_{j}}^{-1}(\alpha) \mathrm{d} \alpha-\sum_{i=1}^{M} \sum_{k=1}^{K} x_{i j k} \leq 0,\right. \\
& \left\{\sum_{i=1}^{M} \sum_{j=1}^{N} x_{i j k}-\int_{0}^{1} \Phi_{\widetilde{V}_{k}}^{-1}(1-\alpha) \mathrm{d} \alpha \leq 0,\right. \\
& \{i=1,2, \ldots, M ; j=1,2, \ldots, N ; k=1,2, \ldots, K
\end{aligned}
$$

where $\Phi_{\tilde{A}_{i}}^{-1}, \Phi_{\widetilde{B}_{j}}^{-1}, \Phi_{\widetilde{V}_{k}}^{-1}, \Phi_{\tilde{\lambda}_{j}}^{-1}, \Phi_{\widetilde{\eta}_{j}}^{-1}, \Phi_{\tilde{c}_{i j k}}^{-1}$, and $\Phi_{\tilde{f}_{i j k}}^{-1}$ are the inverse distributions of uncertain variables $\widetilde{A}_{i}, \widetilde{B}_{j}, \tilde{V}_{i j k}, \tilde{\lambda}_{j}, \widetilde{\eta}_{j}$, $\widetilde{c}_{i j k}$, and $\widetilde{f}_{i j k}$, respectively.

Proof. According to equation (8) and Theorem 2, in the

$$
E\left[\sum_{i=1}^{M} \sum_{j=1}^{N} \sum_{k=1}^{K} \tilde{c}_{i j k} x_{i j k}\right]=\sum_{i=1}^{M} \sum_{j=1}^{N} \sum_{k=1}^{K} x_{i j k} \int_{0}^{1} \Phi_{c_{i j k}}^{-1}(\alpha) \mathrm{d} \alpha
$$

objective function equation (22), we have

$$
\begin{gathered}
E\left(\sum_{i=1}^{M} \sum_{j=1}^{N} \sum_{k=1}^{K} y_{i j k} \tilde{f}_{i j k}\right)=\sum_{i=1}^{M} \sum_{j=1}^{N} \sum_{k=1}^{K} y_{i j k} \int_{0}^{1} \Phi_{\tilde{f}_{i j k}^{-1}}^{-1}(\alpha) \mathrm{d} \alpha \\
E\left[\sum_{i=1}^{M} \sum_{j=1}^{N} \sum_{k=1}^{K} e_{j}\left(\tilde{\lambda}_{j}+\widetilde{\eta}_{j}\right) x_{i j k}\right]=\sum_{i=1}^{M} \sum_{j=1}^{N} \sum_{k=1}^{K}\left[\int_{0}^{1} \Phi_{\tilde{\lambda}_{j}^{-1}}^{-1}(\alpha) \mathrm{d} \alpha+\int_{0}^{1} \Phi_{\widetilde{\eta}_{j}}^{-1}(\alpha) \mathrm{d} \alpha\right] x_{i j k} .
\end{gathered}
$$

With the combination of equations (30)-(32), the transformation of objective function in expect value case is completed.

Also, we can demonstrate that the constraints can be converted as equations (26)-(28) in the same way. Then, Theorem 3 is proved.
Lemma 1. Let $\xi_{1}, \xi_{2}, \ldots, \xi_{n}$ be independent uncertain variables with regular uncertainty distributions $\Phi_{1}, \Phi_{2}, \ldots, \Phi_{n}$, respectively. Assume $f\left(\xi_{1}, \xi_{2}, \ldots, \xi_{n}\right)$ is strictly increasing, then

$$
\mathscr{M}\left\{f\left(\xi_{1}, \xi_{2}, \ldots, \xi_{n}\right) \leq \bar{f}\right\} \geq \alpha
$$


which is equivalent to

$$
f\left(\Phi_{1}^{-1}(\alpha), \ldots, \Phi_{n}^{-1}(\alpha)\right) \leq \bar{f}
$$

where $\alpha$ is a given confidence level.

Theorem 4. Based on the assumptions in Theorem 3, the equivalent expression of the model in equation (24) is

$$
\begin{aligned}
& \left\{\min \left[\Phi_{Z}^{-1}(\alpha)\right],\right. \\
& \left\{\begin{array}{l}
\text { s.t. } \\
\sum_{j=1}^{N} \sum_{k=1}^{K} x_{i j k}-\Phi_{\widetilde{A}_{i}}^{-1}\left(1-\alpha_{i}\right) \leq 0,
\end{array}\right. \\
& \left\{\left[1+\Phi_{\widetilde{\eta}_{i}}^{-1}\left(\beta_{j}\right)\right] \Phi_{\widetilde{B}_{j}}^{-1}\left(\beta_{j}\right)-\sum_{i=1}^{M} \sum_{k=1}^{K} x_{i j k} \leq 0,\right. \\
& \left\{\sum_{i=1}^{M} \sum_{j=1}^{N} x_{i j k}-\Phi_{\widetilde{V}_{k}}^{-1}\left(1-\gamma_{k}\right) \leq 0,\right. \\
& \{i=1,2, \ldots, M ; j=1,2, \ldots, N ; k=1,2, \ldots, K,
\end{aligned}
$$

where

$$
\begin{aligned}
\Phi_{Z}^{-1}(\alpha)= & \sum_{i=1}^{M} \sum_{j=1}^{N} \sum_{k=1}^{K}\left\{\Phi_{c_{i j k}}^{-1}(\alpha) x_{i j k}+y_{i j k} \Phi_{f_{i j k}}^{-1}(\alpha)\right. \\
& \left.+e_{j}\left[\Phi_{\lambda_{j}}^{-1}(\alpha)+\Phi_{\eta_{j}}^{-1}(\alpha)\right] x_{i j k}\right\},
\end{aligned}
$$

is the inverse distribution of $\Phi_{Z}$ and $\alpha, \alpha_{i}, \beta_{j}$, and $\gamma_{k}$ are preset confidence levels.

Proof. Based on Lemma 1, $\mathscr{M}\{Z \leq \bar{Z}\} \geq \alpha$ is equivalent to

$$
\Phi_{Z}^{-1}(\alpha) \leq \bar{Z}
$$

and the objective function in equation (24) can be altered to

$$
\left\{\begin{array}{l}
\min \bar{Z} \\
\text { s.t. } \\
\Phi_{Z}^{-1}(\alpha) \leq \bar{Z},
\end{array}\right.
$$

which can also be converted to

$$
\min \Phi_{Z}^{-1}(\alpha)
$$

Then, due to Theorem 1 and the independence of all the uncertain variables, the expression of $\Phi_{Z}^{-1}(\alpha)$ can be easily inferred as equation (40).

Similarly, the remaining constraints in equation (24) can be converted to equations (36)-(38). Then, the proof of Theorem 4 is eventually finished.

\section{Numerical Experiment}

In order to explicate this kind of FCTP, a numerical experiment is designed in this section. We make an
TABLE 1: Supplies and demands.

\begin{tabular}{lc}
\hline Supplies at origins & Demands at destinations \\
\hline$\widetilde{A}_{1} \sim \mathcal{N}(80,3)$ & $\widetilde{B}_{1} \sim \mathcal{N}(52,1)$ \\
$\widetilde{A}_{2} \sim \mathcal{N}(95,2)$ & $\widetilde{B}_{2} \sim \mathcal{N}(45,2)$ \\
$\widetilde{A}_{3} \sim \mathcal{N}(69,2)$ & $\widetilde{B}_{3} \sim \mathcal{N}(40,1)$ \\
\hline
\end{tabular}

TABLE 2: Return rate and exchange rate.

\begin{tabular}{lc}
\hline Return rate & Exchange rate \\
$\widetilde{\lambda}_{1} \sim \mathscr{L}(0.05,0.07)$ & $\widetilde{\eta}_{1} \sim \mathscr{L}(0.03,0.05)$ \\
$\widetilde{\lambda}_{2} \sim \mathscr{L}(0.06,0.11)$ & $\widetilde{\eta}_{2} \sim \mathscr{L}(0.02,0.06)$ \\
$\widetilde{\lambda}_{3} \sim \mathscr{L}(0.04,0.09)$ & $\widetilde{\eta}_{3} \sim \mathscr{L}(0.04,0.08)$ \\
\hline
\end{tabular}

TABLE 3: The maximum capacity.

$\widetilde{V}_{1} \sim \mathcal{N}(120,1) \quad \widetilde{V}_{2} \sim \mathcal{N}(113,2)$

assumption that there exist three origins, three destinations, and two conveyances (truck and train), and then the uncertain model in equation (19) could be detailed as

$$
\left\{\begin{array}{l}
\min \sum_{i=1}^{3} \sum_{j=1}^{3} \sum_{k=1}^{2}\left[\widetilde{c}_{i j k} x_{i j k}+y_{i j k} \widetilde{f}_{i j k}+e_{j}\left(\widetilde{\lambda}_{j}+\widetilde{\eta}_{j}\right) x_{i j k}\right] \\
\text { s.t. } \\
\sum_{j=1}^{3} \sum_{k=1}^{2} x_{i j k} \leq \widetilde{A}_{i} \\
\sum_{i=1}^{3} \sum_{k=1}^{2} x_{i j k} \geq\left(1+\widetilde{\eta}_{j}\right) \widetilde{B}_{j} \\
\sum_{i=1}^{3} \sum_{j=1}^{3} x_{i j k} \leq \widetilde{V}_{k} .
\end{array}\right.
$$

The parameters are detailed in Tables 1-5.

As the transportation problem constructed in this paper belongs to a NP-hard problem, intelligent algorithms need to be utilized to implement the simulations. For simplicity, three strategies are adopted and tested in Matlab environment. The first one is the traditional interior point method (IPM), and the two other intelligent algorithms are genetic algorithm (GA) and particle swarm optimization algorithm (PSO). The corresponding flow charts of GA and PSO are shown in Figures 1 and 2. Besides, the parameters are set as follows. GA: maxgeneration $=500$, Popsize $=50$, crossover probability $P_{c}=0.8$, and mutation probability $P_{m}=0.2$; PSO: Popsize $=40$, maxgeneration $=1000$, and $c_{1}=c_{2}=2$.

Under the expected value criterion, the optimal results obtained by GA and PSO are displayed in Table 6. Here, "AL" (Algorithm) represents the GA or PSO algorithm, "Optimum solution" explicates the optimal decision variables $x_{i j k}$ that lead to the total minimum transportation cost "OV" (Objective value), and "TA" (Total amount) indicates the whole transportation amount.

The corresponding evolution process of GA and PSO is revealed in Figures 3 and 4, respectively.

We can learn from Table 6 that under the expected value criterion, IPM performs best while PSO loses its advantage. 
TABLE 4: Direct transportation cost $\tilde{c}_{i j k}$ and fixed charge $\tilde{f}_{i j k}$.

\begin{tabular}{|c|c|c|c|c|c|c|c|}
\hline & & & 1 & & & 2 & \\
\hline & & 1 & 2 & 3 & 1 & 2 & 3 \\
\hline & 1 & $\mathscr{L}(0.32,0.41)$ & $\mathscr{L}(0.26,0.31)$ & $\mathscr{L}(0.36,0.52)$ & $\mathscr{L}(0.26,0.48)$ & $\mathscr{L}(0.31,0.35)$ & $\mathscr{L}(0.22,0.28)$ \\
\hline$\widetilde{c}_{i j k}$ & 2 & $\mathscr{L}(0.40,0.46)$ & $\mathscr{L}(0.39,0.55)$ & $\mathscr{L}(0.22,0.30)$ & $\mathscr{L}(0.40,0.55)$ & $\mathscr{L}(0.33,0.40)$ & $\mathscr{L}(0.32,0.37)$ \\
\hline & 3 & $\mathscr{L}(0.30,0.40)$ & $\mathscr{L}(0.30,0.55)$ & $\mathscr{L}(0.42,0.45)$ & $\mathscr{L}(0.27,0.31)$ & $\mathscr{L}(0.23,0.25)$ & $\mathscr{L}(0.40,0.52)$ \\
\hline & 1 & $\mathscr{L}(3,5)$ & $\mathscr{L}(6,9)$ & $\mathscr{L}(7,9)$ & $\mathscr{L}(4,8)$ & $\mathscr{L}(2,7)$ & $\mathscr{L}(4,8)$ \\
\hline$\tilde{f}_{i j k}$ & 2 & $\mathscr{L}(5,6)$ & $\mathscr{L}(8,10)$ & $\mathscr{L}(6,10)$ & $\mathscr{L}(6,10)$ & $\mathscr{L}(3,9)$ & $\mathscr{L}(5,6)$ \\
\hline & 3 & $\mathscr{L}(5,7)$ & $\mathscr{L}(6,7)$ & $\mathscr{L}(7,10)$ & $\mathscr{L}(5,8)$ & $\mathscr{L}(5,6)$ & $\mathscr{L}(7,9)$ \\
\hline
\end{tabular}

TABLE 5: Direct transportation cost $e_{j}$ back to the manufacture.

Transportation cost

$e_{1}=0.25$

$e_{2}=0.31$

$e_{3}=0.42$

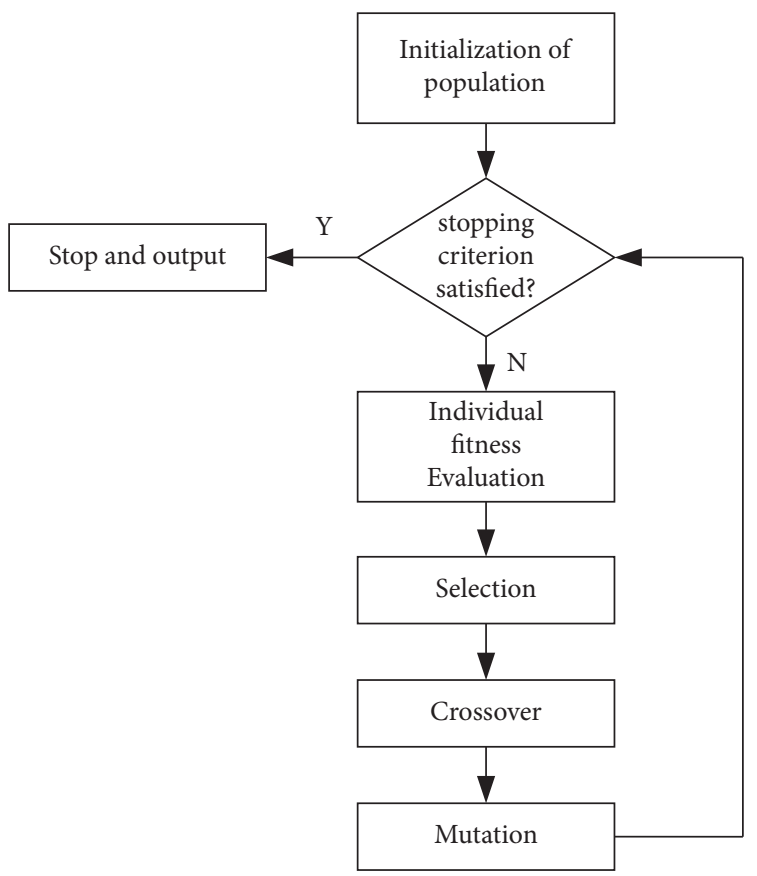

Figure 1: Flow chart of GA.

To reveal their effectiveness further, the three algorithms are tested for the chance-constrained case with different confidence levels. For simplicity, $\alpha, \alpha_{i}, \beta_{j}$, and $\gamma_{k}$ are deemed to be identical, and the optimal results for the three methods are elaborated in Table 7.

Table 7 reveals that IPM is prior in this situation, which is in accordance with the result for the expected value model case. Besides, with the decrement of $\alpha$, the total transportation cost shows a decreasing trend as well. The reason is that as the confidence level decreases, the corresponding uncertain variable's value lowers too, leading to less total transportation cost. More simulations under different

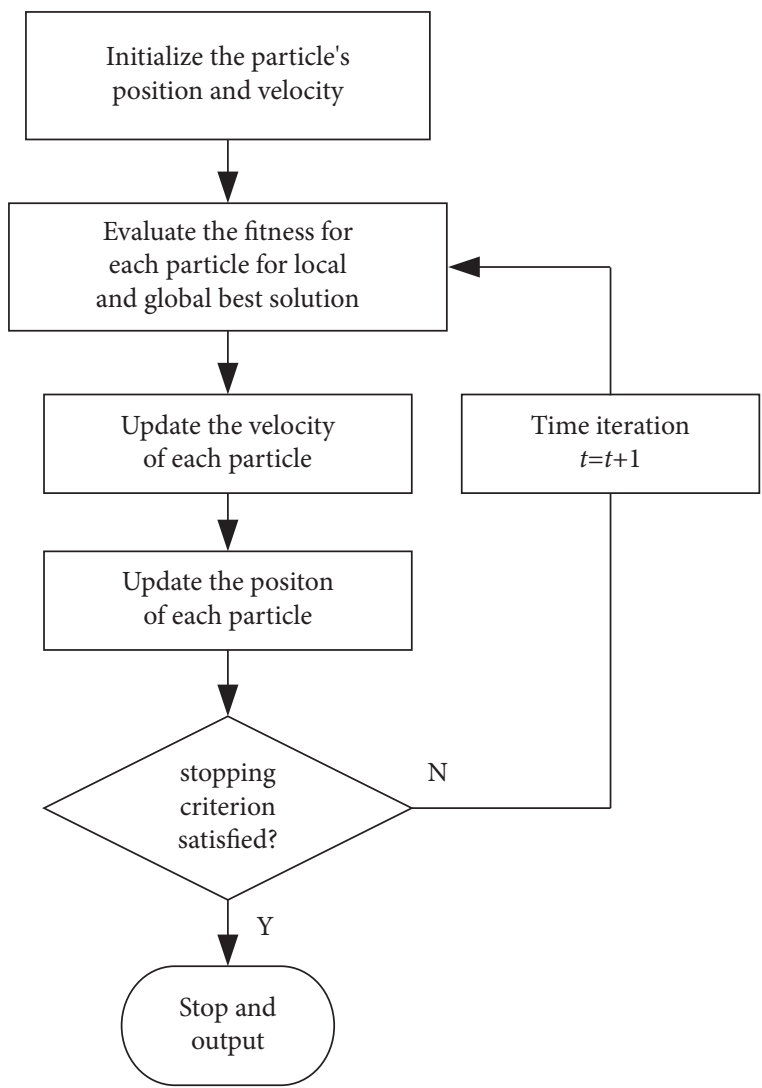

FIgURE 2: Flow chart of PSO.

confidence levels are implemented for the chance-constrained case, with the sensitivity analysis explicated in Figure 5 .

Figure 5 illustrates that the objective value reveals increasing trend with the increment of confidence level $\alpha$. For the chance-constrained model, its feasible domain will alter according the confidence level and the higher confidence level means more requirements should be satisfied, leading to higher transportation cost. This trend also obeys the common sense because when the constraints become more elastic, less cost is required, which is also reflected in the simulation. Besides, the optimal results gained via IPM are always most competitive, which is similar to the previous conclusion. To verify their effectiveness in a 
TABLE 6: Optimal results of the expected value model.

\begin{tabular}{lccc}
\hline AL & OV & TA & Optimum solution \\
\hline IPM & \multirow{2}{*}{144.7967} & 146.4981 & $10.4891,10.3917,13.7854,9.2263,0,16.6921,5.1146,0.4321,0$ \\
& & $6.7833,17.1350,8.7592,11.6155,17.0715,1.1317,17.8708,0,0$ \\
GA & \multirow{2}{*}{165.3065} & $0.0120,9.5263,7.8053,7.8889,8.0551,13.8736,9.5404,9.0114,16.0030$ \\
& & $0,0,6.6735,9.0118,9.0116,9.1580,8.3339,6.6843,7.3004$ \\
PSO & \multirow{2}{*}{173.6376} & \multirow{2}{*}{146.4986} & $9.0461,13.5694,26.2959,10.0123,4.5613,7.7789,1.0765,8.1923,0.6276$ \\
& & $0,10.7862,9.8593,2.8752,20.3554,6.4939,5.3677,2.4219,7.1787$ \\
\hline
\end{tabular}

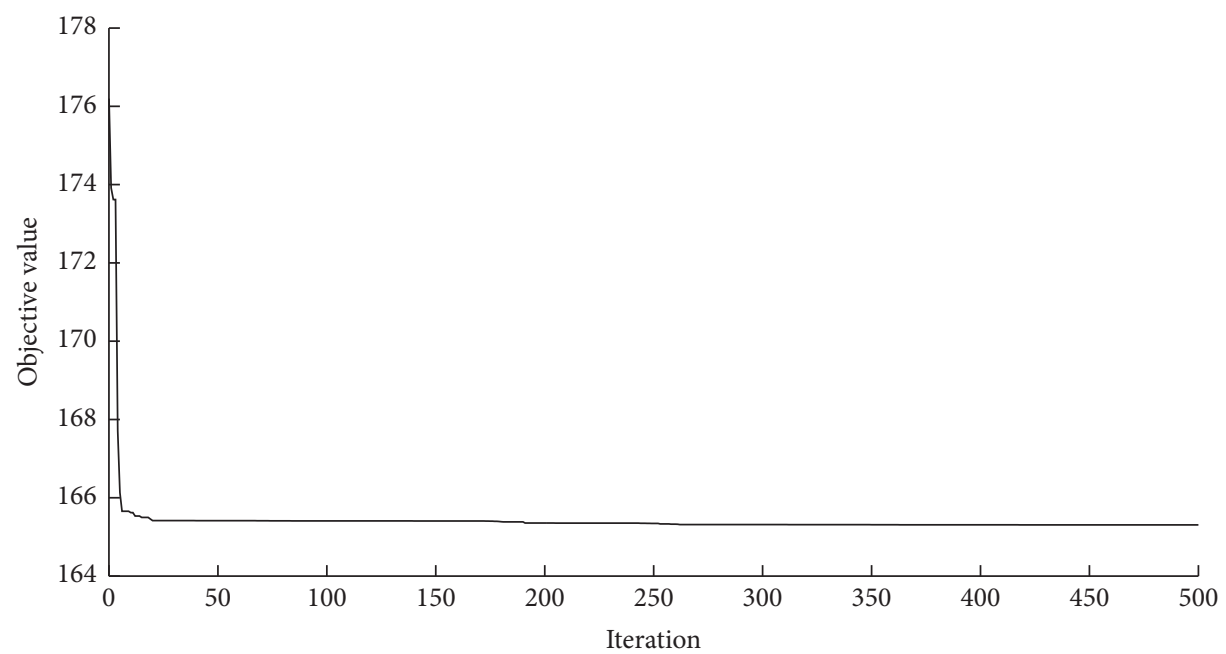

FIgURE 3: The evolution process of GA.

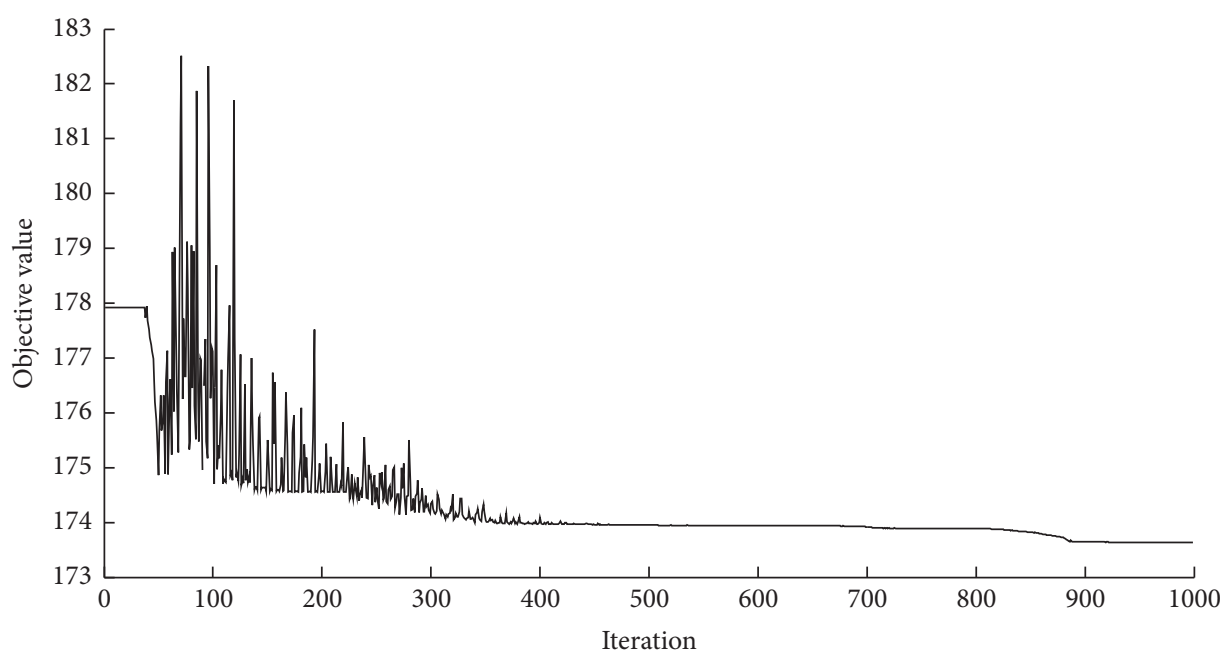

Figure 4: The evolution process of PSO. 
TABLe 7: Optimal results of the chance-constrained model.

\begin{tabular}{|c|c|c|c|c|}
\hline$\alpha$ & $\mathrm{AL}$ & $\mathrm{OV}$ & TA & Optimum solution \\
\hline \multirow{3}{*}{0.95} & IPM & 173.9528 & 152.1659 & $\begin{array}{c}11.8470,6.0939,14.2020,10.9980,0,18.2653,6.2078,0,0 \\
5.9004,16.0804,9.1164,11.8861,20.2160,0,19.9447,1.4078,0\end{array}$ \\
\hline & GA & 203.3171 & 152.2629 & $\begin{array}{c}10.2387,0,8.2735,8.2736,7.3241,7.3596,10.0567,13.4528,8.2735 \\
9.1262,7.3240,7.3770,8.9023,13.6982,8.8244,8.2733,7.8315,7.6536\end{array}$ \\
\hline & PSO & 205.1389 & 152.2100 & $\begin{array}{c}6.7949,8.4686,17.6868,8.7530,3.0638,9.5009,6.0860,8.9347,0 \\
10.4751,10.5855,8.0048,9.7952,16.1715,0.0316,14.0991,7.1646,6.5939\end{array}$ \\
\hline \multirow{3}{*}{0.9} & IPM & 169.1728 & 150.1875 & $\begin{array}{c}11.5477,6.3658,13.2551,11.2050,0,15.9906,6.3572,0,0 \\
7.4859,15.4963,9.7381,11.9720,19.5227,0,18.1324,3.1183,0\end{array}$ \\
\hline & GA & 197.7742 & 150.1851 & $\begin{array}{c}8.9181,0,7.5977,7.5987,7.2601,6.9119,8.9181,9.1070,8.8469 \\
7.5987,6.9122,8.3678,8.9181,19.9036,8.0825,10.3531,7.3877,7.5028\end{array}$ \\
\hline & PSO & 198.7735 & 150.1875 & $\begin{array}{l}8.5525,6.8108,17.2381,10.1274,6.5618,14.0327,5.7079,8.7489,0 \\
7.6798,17.1644,6.2950,9.8664,16.0792,0,15.03320 .2170,0.0725\end{array}$ \\
\hline \multirow{3}{*}{0.85} & IPM & 162.0722 & 148.8783 & $\begin{array}{l}11.0557,6.8205,13.7784,10.6551,0,15.7933,7.0420,1.1576,0 \\
7.4030,15.1529,9.2647,11.7167,17.6529,0,17.6095,3.7762,0\end{array}$ \\
\hline & GA & 193.0846 & 148.8761 & $\begin{array}{c}8.2291,9.5366,7.7029,8.5107,7.0891,7.6889,10.6834,8.9365,0 \\
9.3323,7.7720,7.2546,9.8298,8.2291,15.4059,8.4937,6.7206,7.4610\end{array}$ \\
\hline & PSO & 196.3841 & 148.8784 & $\begin{array}{c}11.1494,8.2094,8.6612,7.7997,5.8796,16.0841,8.8765,6.3582,0 \\
7.3664,17.0024,4.3000,10.1840,10.6677,7.1591,18.4595,0.7094,0.0116\end{array}$ \\
\hline
\end{tabular}

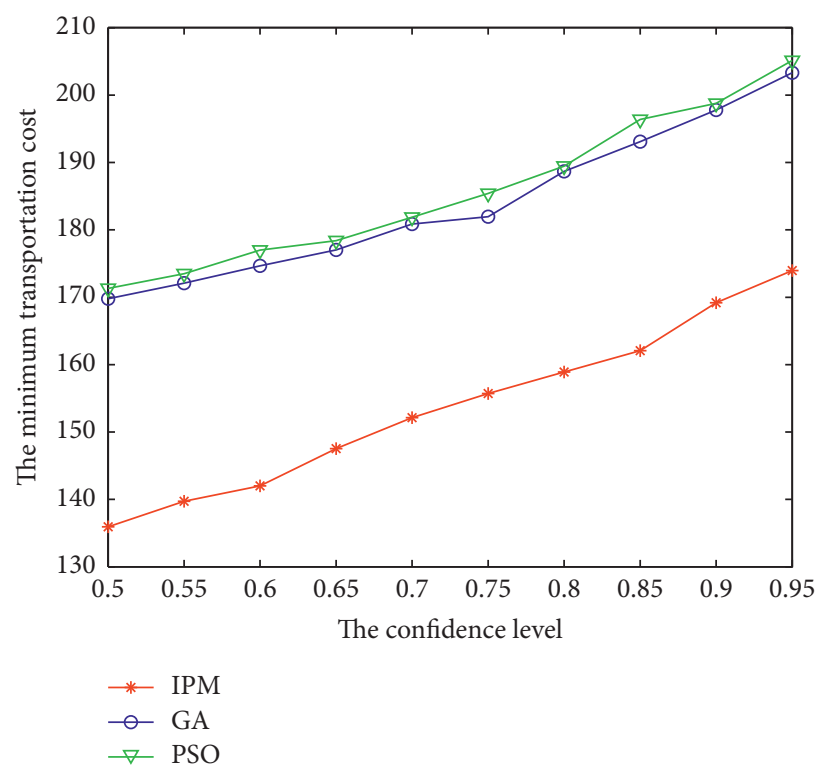

FIGURE 5: Sensitivity analysis of the chance-constrained model.

relatively complex situation, we add a nonlinear factor to the model in equation (44) where the objective function is modified to

$$
\min \sum_{i=1}^{3} \sum_{j=1}^{3} \sum_{k=1}^{2}\left[\widetilde{c}_{i j k} x_{i j k}^{2}+y_{i j k} \tilde{f}_{i j k}+e_{j}\left(\widetilde{\lambda}_{j}+\widetilde{\eta}_{j}\right) x_{i j k}\right],
$$

while other parameters or settings keep unchanged. For the chance-constrained case, the optimal values gained via the three methods are explicated in Figure 6.

The simulation results displayed in 6 demonstrate that IPM still performs best in the nonlinear situation. The above various simulation results indicate the effectiveness of the different solving algorithms in searching for the optimal 


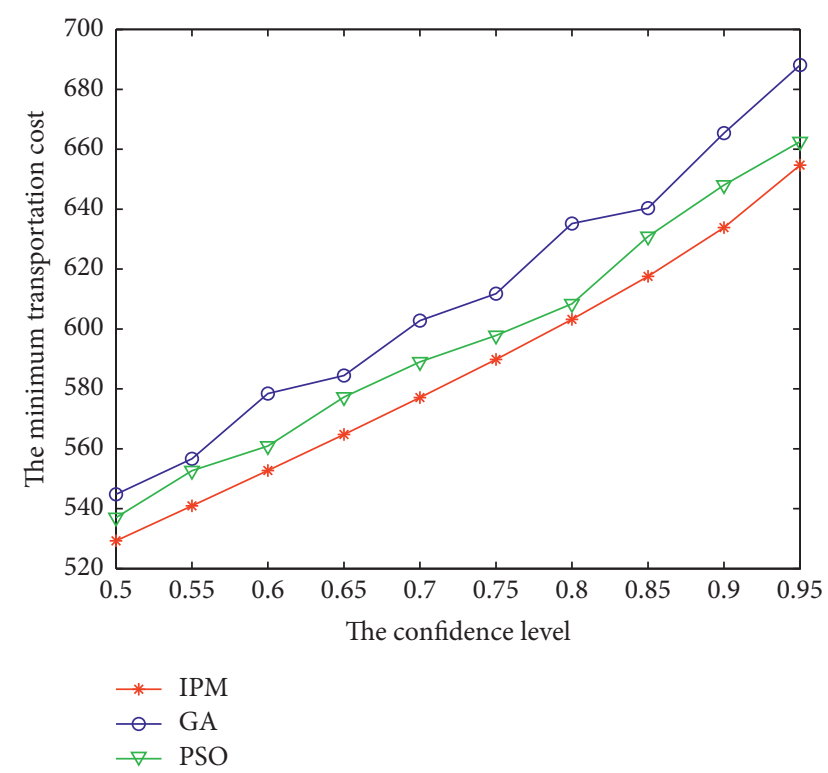

Figure 6: Sensitivity analysis of the chance-constrained model with nonlinear factor.

solutions, making this problem applicable in the decisionmaking scenario.

\section{Conclusions}

This paper studies an uncertain FCTP in which after-sale service as return and exchange policies are guaranteed to meet the customer's demand and raise the service quality. To make the problem more realistic, some indeterministic factors such as supplies, demands, and capacities are considered. When the sample size is large enough, it is possible for us to believe that the estimated probability distribution is close enough to the long-run cumulative frequency. However, when sufficient historical data are unavailable, uncertainty theory is the best option. Based on uncertainty theory, some parameters are supposed to follow uncertain distributions. Then, the basic model is evolved to two other uncertain models in the expected value criterion and chanceconstrained criterion, respectively, and their deterministic forms are achieved via uncertainty inverse distribution method. After that, three algorithms such as IPM, GA, and PSO are utilized to solve the numerical examples, and the corresponding results are displayed in figures. Besides, a modified objective function is proposed for further tests of the three solving methods, and corresponding sensitivity analyses are made. The comparison results reveal that the IPM method provides best performance while the others are less competitive. Moreover, in chance-constrained case, the objective function is nondecreasing with increment of confidence levels, which is in accordance with the theoretical analysis.

Compared with the fixed charge transportation problem in this paper, more aspects could be considered to make it more realistic. For example, product damage may occur during the transportation and it varies a lot according to the product variety. To meet the demand, more products should be conveyed. Also, more constraints such as time limit, time window, and price limitation can be supplemented, which are common in daily transportation and trade. From another point of view, profit-oriented problem is a nice topic. For example, for a particular product, there are always different brands with several different packaging amounts, and the prices are relative to the quality. For the customers, their special demands and purchasing powers will lead to different options, so how to make a proper schedule to maximize the profit is a challenge. Simultaneously, customers are generally price-sensitive, and a transportation problem with two decision variables can be studied.

Based on the study of this paper, more applications could be considered. In task allocation, the same method can be utilized to minimize the total cost. When disassembling some equipment, how to make a proper disassembly process to save time is a challenge. When indeterminacy is put into consideration, all the problems related to schedule or plan can be dealt in a similar way. Also, multiobjective function with more constraints can be studied to make the problem more applicable, providing a more effective way for the decision maker.

\section{Data Availability}

The data used to support the findings of this study are included within the article.

\section{Conflicts of Interest}

The authors declare that they have no conflicts of interest.

\section{Acknowledgments}

This work was supported by the Natural Science Foundation of the Jiangsu Higher Education Institutions of China (20KJB520015) and Changzhou Science \& Technology Program (CJ20200039).

\section{References}

[1] F. L. Hitchcock, "The distribution of a product from several sources to numerous localities," Studies in Applied Mathematics, vol. 20, no. 1-4, pp. 224-230, 1941.

[2] T. C. Koopmans, "Optimum utilization of the transportation system,” Econometrica, vol. 17, pp. 136-146, 1949.

[3] H. Arsham and A. B. Kahn, "A simplex-type algorithm for general transportation problems: an alternative to steppingstone," Journal of the Operational Research Society, vol. 40, no. 6, Article ID 581C590, 1973.

[4] L. Cooper and M. W. Cooper, "An exact algorithm for the concave transportation problem," Computers \& Mathematics with Applications, vol. 2, no. 1, pp. 49-61, 1976.

[5] S. K. Das, A. Goswami, and S. S. Alam, "Multiobjective transportation problem with interval cost, source and destination parameters," European Journal of Operational Research, vol. 117, no. 1, pp. 100-112, 1999.

[6] Y. P. Aneja and K. P. K. Nair, "Bicriteria transportation problem," Management Science, vol. 25, no. 1, pp. 73-78, 1979.

[7] M. Sun, "The transportation problem with exclusionary side constraints and two branch-and-bound algorithms," 
European Journal of Operational Research, vol. 140, no. 3, pp. 629-647, 2002.

[8] W. M. Hirsch and G. B. Dantzig, "The fixed charge problem," Naval Research Logistics Quarterly, vol. 15, no. 3, pp. 413-424, 1968.

[9] P. Robers and L. Cooper, "A study of the fixed charge transportation problem," Computers \& Mathematics with Applications, vol. 2, no. 2, pp. 125-135, 1976.

[10] A. A. Farley and K. V. Richardson, "Fixed charge problems with identical fixed charges," European Journal of Operational Research, vol. 18, no. 2, pp. 245-249, 1984.

[11] S. Sagratella, M. Schmidt, and N. Sudermann-Merx, "The noncooperative fixed charge transportation problem," European Journal of Operational Research, vol. 284, no. 1, pp. 373-382, 2020.

[12] V. Adlakha and K. Kowalski, "A simple heuristic for solving small fixed-charge transportation problems," Omega, vol. 31, no. 3, pp. 205-211, 2003.

[13] H. I. Calvete, C. Galé, J. A. Iranzo, and P. Toth, "A matheuristic for the two-stage fixed-charge transportation problem," Computers \& Operations Research, vol. 95, pp. 113-122, 2018.

[14] A. Biswas, A. A. Shaikh, and S. T. A. Niaki, "Multi-objective non-linear fixed charge transportation problem with multiple modes of transportation in crisp and interval environments," Applied Soft Computing, vol. 80, pp. 628-649, 2019.

[15] L. Yang and Y. Feng, "A bicriteria solid transportation problem with fixed charge under stochastic environment," Applied Mathematical Modelling, vol. 31, no. 12, pp. 2668-2683, 2007.

[16] L. Bertazzi and F. Maggioni, "A stochastic multi-stage fixed charge transportation problem: worst-case analysis of the rolling horizon approach," European Journal of Operational Research, vol. 267, no. 2, pp. 555-569, 2018.

[17] L. A. Zadeh, "Fuzzy sets," Information and Control, vol. 8, no. 3, pp. 338-353, 1965.

[18] S. Majumder, S. Kar, and T. Pal, "Rough-fuzzy quadratic minimum spanning tree problem," Expert Systems, vol. 36, no. 2, Article ID e12364, 2019.

[19] M. B. Kar, S. Kar, S. Guo, X. Li, and S. Majumder, "A new biobjective fuzzy portfolio selection model and its solution through evolutionary algorithms," Soft Computing, vol. 23, no. 12, pp. 4367-4381, 2019.

[20] L. Yang and L. Liu, "Fuzzy fixed charge solid transportation problem and algorithm," Applied Soft Computing, vol. 7, no. 3, pp. 879-889, 2007.

[21] S. Ghosh, S. K. Roy, and A. Ebrahimnejad, "Multi-objective fully intuitionistic fuzzy fixed-charge solid transportation problem," Complex \& Intelligent Systems, vol. 7, Article ID 1009C1023, 2021.

[22] S. Halder, B. Das, G. Panigrahi, and M. Maiti, "Some special fixed charge solid transportation problems of substitute and breakable items in crisp and fuzzy environments," Computers \& Industrial Engineering, vol. 111, pp. 272-281, 2017.

[23] S. Golmohamadi, R. Tavakkoli-Moghaddam, and M. Hajiaghaei-Keshteli, "Solving a fuzzy fixed charge solid transportation problem using batch transferring by new approaches in meta-heuristic," Electronic Notes in Discrete Mathematics, vol. 58, pp. 143-150, 2017.

[24] B. Liu, Uncertainty Theory, Springer-Verlag, Berlin, Germany, 2nd edition, 2007.

[25] B. Liu, Uncertainty Theory: A Branch of Mathematics for Modeling Human Uncertainty, Springer-Verlag, Berlin, Germany, 2007.
[26] Z. Lu, Y. Zhu, and B. Li, "Critical value-based Asian option pricing model for uncertain financial markets," Physica A: Statistical Mechanics and Its Applications, vol. 525, pp. 694-703, 2019.

[27] K. Zhu, J. Shen, and X. Yao, "A three-echelon supply chain with asymmetric information under uncertainty," Journal of Ambient Intelligence and Humanized Computing, vol. 10, no. 2, pp. 579-591, 2019.

[28] Y. Lan, J. Peng, F. Wang, and C. Gao, "Quality disclosure with information value under competition," International Journal of Machine Learning and Cybernetics, vol. 9, no. 9, pp. 1489-1503, 2018.

[29] J. Shen and K. Zhu, "An uncertain single machine scheduling problem with periodic maintenance," Knowledge-Based Systems, vol. 144, no. 15, pp. 32-41, 2018.

[30] S. Majumder, B. Saha, P. Anand, S. Kar, and T. Pal, "Uncertainty based genetic algorithm with varying population for random fuzzy maximum flow problem," Expert Systems, vol. 35, no. 4, Article ID e12264, 2018.

[31] D. Mou, W. Zhao, and X. Chang, "A transportation problem with uncertain truck times and unit costs," Industrial Engineering \& Management Systems, vol. 12, no. 12 , pp. 30-35, 2013.

[32] L. Chen, J. Peng, and B. Zhang, "Uncertain goal programming models for bicriteria solid transportation problem," Applied Soft Computing, vol. 51, pp. 49-59, 2017.

[33] S. Majumder, P. Kundu, S. Kar, and T. Pal, "Uncertain multiobjective multi-item fixed charge solid transportation problem with budget constraint," Soft Computing, vol. 23, no. 10, pp. 3279-3301, 2019.

[34] Y. Sheng and K. Yao, "Fixed charge transportation problem and its uncertain programming model," Industrial Engineering and Management Systems, vol. 11, no. 2, pp. 183-187, 2012.

[35] L. Liu, B. Zhang, and W. Ma, "Uncertain programming models for fixed charge multi-item solid transportation problem," Soft Computing, vol. 22, no. 17, pp. 5825-5833, 2018.

[36] J. Shen and K. Zhu, "An uncertain two-echelon fixed charge transportation problem," Soft Computing, vol. 24, no. 5, pp. 3529-3541, 2019.

[37] J. Roy, S. Majumder, S. Kar, and K. Adhikary, "A multiobjective multi-product solid transportation model with rough fuzzy coefficients," International Journal of Uncertainty, Fuzziness and Knowledge-Based Systems, vol. 27, no. 5, pp. 719-753, 2019.

[38] Y. Gao and S. Kar, "Uncertain solid transportation problem with product blending," International Journal of Fuzzy Systems, vol. 19, no. 6, pp. 1916-1926, 2017.

[39] Y. Sheng and S. Kar, "Some results of moments of uncertain variable through inverse uncertainty distribution," Fuzzy Optimization and Decision Making, vol. 14, no. 1, pp. 57-76, 2015. 